\title{
6. Die Verleger Carl Gottlob Beck und Carl Heinrich Beck
}

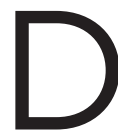

er Nördlinger Ratskonsulent Anton Jakob

Dolp charakterisierte Beck in der Aus-

einandersetzung mit Wekhrlin als einen

Nun aufwärts froh den Blick gewandt und vorwärts fest den Schritt. August Hermann Francke «fleißigen, bestrebsamen, ordentlichen, rechtschaffenen, seinen Geschäften treu abwartenden - alle Tücke und Hinterlist verabscheuenden, friedliebenden, auch wohlhabenden Mann». Aber was darf man auf eine solche Charakterisierung in einem Rechtshandel geben? Verwies doch sein Gegner, Wekhrlin, auf die «landkundigen Grobheiten» und auf «vielerlei mitgespielte Betrügereien». So habe Beck zweimal ohne sein Wissen den «Rabiosus» nachgedruckt, Briefe unterschlagen, sich an Verträge nicht gehalten und finanzielle Zusagen nicht eingehalten. Mehr noch: Beck liege mit seinen Kunden im Streit wegen falscher Abrechnungen und schuldig gebliebener Lieferungen. ${ }^{1}$ Und es stellt sich dieselbe Frage: Welchen Quellenwert hat solch eine Aussage aus einem Rechtsstreit, will man sich über den Charakter einer Partei Klarheit verschaffen?

\section{Carl Gottlob Beck}

Carl Gottlob Beck hat uns keine Selbstzeugnisse hinterlassen. Seine umfangreiche Korrespondenz ist nicht erhalten. Nur schemenhaft lässt sich seine Persönlichkeit ausmachen. Und wenn man die Lehren der Individualpsychologie ernst nimmt, so darf man sich auch die Frage vorlegen, wie nah man in der Beschreibung einem Menschen überhaupt kommen mag, der kein Zeitgenosse ist und von dem man sich keinen unmittelbaren Eindruck verschaffen kann. Immerhin: Eine feste Burg war ihm der Glaube seiner Väter, aber als Verleger handelte er nie dogmatisch, sondern flexibel, bisweilen durchaus risikofreudig. Man ist geneigt, in sein Profil mit den scharfen Gesichtszügen einen selbstbewussten Spötter hineinzulesen, der bisweilen sein Gegenüber vor den Kopf stieß und zu seiner Umwelt auf Distanz blieb. Sein «straff gebundenes Perückenzöpfchen», um 
Albrecht Schöne zu zitieren, bedeckte einen «eisernen Nacken», der vielleicht erahnen lässt, wie unerbittlich Beck seine Interessen verfolgen konnte. ${ }^{2}$

Carl Gottlob Beck stand keinem großen Buchhandelsunternehmen vor. ${ }^{3}$ Andere Unternehmen gaben damals in Deutschland den Ton an: die 1659 gegründete Cotta'sche Verlagsbuchhandlung in Tübingen (seit 1810 in Stuttgart ansässig), die Nicolaische Verlagsbuchhandlung, die 1713 in Berlin ihre Pforten öffnete, und die 1785 gegründete G. J. Göschen'sche Verlagsbuchhandlung in Leipzig. Becks Geschäft konnte man auch nicht mit Friedrich Perthes vergleichen, der in Hamburg die erste Sortimentsbuchhandlung gegründet haben soll. ${ }^{4}$ Mithin war er keiner der führenden Verleger der Aufklärung, deren Produkte sich zu Dutzenden oder sogar zu Hunderten im Lagerkatalog von Friedrich Nicolai aus dem Jahr 1787 finden. Ebendieser Katalog nennt auf den ersten drei Plätzen den Verlagsort Leipzig mit 1321 Titeln, dann Berlin (859) und Halle (187). Nördlingen zählte mit Chemnitz, Nordhausen und Salzburg zu den Orten, an denen jeweils ein Verlag je drei Titel zum Kauf anbot. Während sich in dem Katalog 25 Verlage in Berlin nachweisen lassen und 24, in Leipzig, zählte Nördlingen zu den fast 50 Orten, die nur mit einem Verlag vertreten waren. ${ }^{5}$ Aber in der buchhändlerischen Provinz zog Beck Ansehen und Gewinn daraus, dass er der einzige Buchdrucker in der Reichsstadt Nördlingen war und sich nicht gegen einen oder mehrere Konkurrenten durchsetzen musste.

Entscheidend für den wirtschaftlichen Erfolg war die Verankerung des Geschäfts im lokalen und regionalen Umfeld. Nur hier konnten sichere Einkünfte erzielt werden. Kirche und Schule garantierten Prosperität. $177^{2}$ sicherte ihm der Kauf der fürstlich wallersteinischen Buchdruckerei das Monopol auf die Gesang- und Schulbücher, die auf diesem Territorium eingeführt wurden. So druckte und vertrieb Carl Gottlob Beck 1783 das neue Evangelische Gesangbuch Nördlingens. Am 15.August desselben Jahres wurde es im Nördlinger Wochenblatt ausführlich angekündigt und die Initiative des «Hochedlen und Hochweisen Magistrats» gerühmt, durch dieses neue Gesangbuch, das «mit dem Advent dieses Jahrs zum öffentlichen Gebrauch in den hiesigen Kirchen und Schulen eingeführt werden» solle, «wahre christliche Erbauung» zu befördern und zu größerer «Gleichförmigkeit des öffentlichen Gottesdiensts» zu gelangen. ${ }^{6}$ Die wichtigsten Einnahmequellen waren nicht gelehrte Publikationen, sondern vielmehr Erbauungs- und Gebetbücher, vielerlei Ratgeberliteratur, Kalender und Reden, Verordnungen und Flugschriften sowie Zeitungen. Dies war durchaus zeittypisch, wie sich einer der Lehrlinge des alten Frankfurter Druck- 
und Verlagshauses H. L. Brönner für die Zeit um 1800 erinnerte: «Die Pressen der Brönner'schen Officin waren nämlich fortwährend mit Bibeln, Testamenten, Psaltern, Katechismen, Gebet- und Gesangbüchern usw. beschäftigt, in deren Absatz im Großen das Brünnchen zu finden war, was das Becken der Sparsamkeit nach und nach so lange füllen half.» ${ }^{7}$

Carl Gottlob Beck vertrat in seinen Verlagsprodukten aufgeklärte Meinungen. Aber die Vision eines gar herrschaftsfreien Miteinanders der Menschen hegte der Protestant Beck nicht. Wenn in seiner Zeitschrift Positionen vertreten wurden, die er nicht teilte, so scheute er sich nicht, im Namen der Redaktion Widerspruch zu formulieren. Als ein Beiträger Kritik an der Todesstrafe übte, distanzierte sich Carl Gottlob Beck von diesem Artikel: «Der Herr Einsender überzeugt mich nicht; denn unter der Menge von Zuschauern sind doch gewiss einige, auf welche die öffentlichen Hinrichtungen Eindruck machen, und da Bösewichte größtenteils Poltrons sind, so kann es nicht fehlen, dass nicht hier und dort ein Verbrechen verhütet werde.» ${ }^{8}$

Wenn man die von Reinhard Wittmann für die Goethezeit vorgeschlagene Verlegertypologie, die zwischen Fabrikanten, ehrlichen Maklern und Provinzverlegern unterscheidet, anwenden will, war Carl Gottlob Beck kein «Buchfabrikant» wie Engelhard Benjamin Schwickert oder Johann Friedrich Weygand in Leipzig, die Immanuel Kant in seiner Polemik gegen den Verleger Friedrich Nicolai treffend charakterisierte: «Ein erfahrner Kenner der Buchmacherei wird als Verleger nicht erst darauf warten, dass ihm von schreibseligen, allezeit fertigen Schriftstellern ihre eigene Ware zum Verkauf angeboten wird; er sinnt sich als Director einer Fabrik die Materie sowohl als die Facon aus, welche mutmaßlich [...] die größte Nachfrage, oder allenfalls auch nur die schnellste Abnahme haben wird.»9 Beck war insofern ein «Provinzverleger», als er in der Provinz wirkte, aber er bewährte sich auch als «ehrlicher Makler», der durchaus nicht nur an seinen eigenen Profit, sondern auch, wie seine Verbindung zu Wekhrlin gezeigt hat, an die Interessen seiner Autoren dachte. ${ }^{10}$ Zweifelhafte Geschäftspraktiken wie Raubdrucke waren ihm nicht fremd, und er wandte sie an, wenn es darum ging, Geld zu verdienen. Er hatte ein feines Gespür für attraktive Themen und Formate, und er verstand es, durch die Pflege seiner Netzwerke Autoren an seinen Verlag zu binden.

Carl Gottlob Beck war zugleich ein erfolgreicher Stadtbuchhändler und -drucker. Die Buchhandlung und Druckerei in Nördlingen deckten vor Becks Übernahme nur den Bedarf der Reichsstadt. Becks offensive Politik erweiterte den Kreis seiner Kunden. Der Verleger machte Geschäfte mit «großen Herren», mit weltlichen und geistlichen Fürsten in der Nachbar- 
schaft, denen er die Novitäten persönlich vorbeigebracht haben wird, aber er war auch ein Buchhändler für «Bürger und Mittelstand», deren Interessen sein breites Sortiment widerspiegelte. ${ }^{11}$ Die enge Verbindung sowohl zum städtischen Bürgertum als auch zu den umliegenden Abteien, Klöstern und souveränen Häusern war die Grundlage für seinen wirtschaftlichen Erfolg. Seine Bücher verkaufte er auch in anderen Gebieten des Reiches, vor allem im benachbarten Württemberg. Von dort kam in den $1780 e r$ Jahren der Buchdruckergeselle Johann Gottfried Vogel, der ihm zur Hand ging. ${ }^{12}$ Beck erzielte mit seinem Programm überregionalen Erfolg, wie allein schon die Tatsache zeigt, dass seine Bücher in Friedrich Nicolais Lagerkatalog aus dem Jahr 1787 Aufnahme fanden und in der Jenaer «Allgemeinen Literatur-Zeitung», aber auch in anderen literaturkritischen Journalen wie in der «Neuen allgemeinen deutschen Bibliothek» Friedrich Nicolais oder in Isaak Iselins «Ephemeriden der Menschheit» besprochen wurden. ${ }^{13}$

Die Trennung von Druck, Verlag und Sortiment, die die sukzessive Entstehung eines Zwischenbuchhandels ermöglichte, war damals noch unüblich und in einer Kleinstadt wie Nördlingen ohnehin nicht möglich. Wie in vielen anderen vergleichbaren Orten blieben die verschiedenen Sparten des Literaturbetriebs in einer Hand: Carl Gottlob Beck war Verleger, Drucker und Händler und vereinigte in seiner Person das herstellende und das vertreibende Gewerbe. Beck stand auch als Lektor, wie wir heute sagen würden, seinen Autoren zur Seite. Johann August Philipp Gesner bemerkte in der Vorrede zu seinen «Entdeckungen der neuesten Zeit in der Arzneygelahrtheit»: «Die Korrektur des Drucks hat Herr Beck ganz allein, und, wie ich sehe, wohl besorgt. Ich finde keine erheblichen und überhaupt wenige Druckfehler.» ${ }^{14}$

Carl Gottlob Beck verstand seinen Beruf in protestantischer Tradition als Berufung. War er ein Mann des Geldes oder ein Mann des Geistes, ein Aufklärer oder ein Kaufmann? Zwar gab es kein Literaturcafé und keinen bürgerlichen Salon, aber immerhin richtete Beck, wie bereits erwähnt, eine Lesegesellschaft und eine Leihbibliothek ein und gab eine Zeitung heraus. Der Spötter Wekhrlin, der mittlerweile in Nürnberg lebte, meinte, die Langeweile in Nördlingen bringe Carl Gottlob Beck fast um. Ohne den Zuspruch der Auswärtigen hätte er verhungern müssen. ${ }^{15}$ Er wird nicht bestritten haben, dass sein Betrieb in der Provinz lag; aber der Schriftsteller Karl Gutzkow bemerkte 1837 pointiert: «Wir leben überall in der Provinz.» ${ }^{16}$ Am Beispiel von Carl Gottlob Beck und seiner Buchdruckerei und Buchhandlung kann exemplarisch gezeigt werden, wie die vielzitierte Leserevolution und «Volksaufklärung» in kleinen Städten verlief. Eine 
wachsende Leserschaft, die von der verbesserten Bildungssituation profitierte, begnügte sich auch in einer kleinen Reichsstadt nicht länger nur mit Gesangbüchern und Kalendern. Beck veränderte das kulturelle Profil der Stadt, wie ein Zeitgenosse hervorgehoben hat, dessen Urteil schon deshalb unverdächtig ist, da er es in einer Polemik gegen eine von Beck verlegte Schrift äußerte. «Nördlingen», so schrieb der aus der Reichsstadt stammende kurmainzische Geheimrat Georg Wilhelm Zapf, «hat in älteren Zeiten in bezug auf die Gelehrsamkeit nicht die allergeringsten Vorzüge und von Verdiensten lässt sich gar nicht reden. Dieser Ort glich einer dickfinsteren Nacht mit dunkelschwarzen Wolken behangen, aus denen manchmal ein ganz schwacher Blitz hervordrang, sich aber gleich wieder verhüllte. Erst in der neueren Zeit macht die Stadt eine gewisse merkwürdige Periode in der Gelehrsamkeit durch [...]. Die Buchdruckerei und der Buchhandel wurden durch die unermüdlichen Bemühungen eines Beck lebhafter; er regte zu Unternehmungen, zu gelehrten Arbeiten an, da wo vorher alles tot und ausgestorben war.» ${ }^{17}$

Carl Gottlob Beck machte bald guten Umsatz. Fünfzehn Jahre nach der Übernahme der Mundbach'schen Druckerei, am zo. März 1778, erwarb er für 2800 Gulden von der Witwe des Bürgermeisters Daniel Eberhard Dolp in der Bergergasse 3 ein ansehnliches Anwesen, das «aus einem stattlichen, im siebzehnten Jahrhundert erbauten zweistöckigen, mit zwei Erkertürmen gezierten Wohnhaus nebst zwei anstoßenden, mit dem Vorderhaus verbundenen, im rechten Winkel zueinanderstehenden mehrgeschossigen Rückgebäuden» bestand. Hinzu kam ein großer Garten. Im Erdgeschoss des Vorderhauses richtete Beck die Buchhandlung ein; die rasch wachsende Familie des Besitzers lebte in den beiden oberen Stockwerken des Vorderhauses. ${ }^{18}$ In einem der Rückgebäude fanden die Setzerei und Druckerei «nebst den dazu gehörigen Nebenräumen», d.h. dem Papierlager, der Walzengießerei und dem Waschraum, ihr Unterkommen. Dort wohnten auch die Gehilfen, Lehrlinge und Gesellen. Das Gesinde war bis ins 19. Jahrhundert integraler Bestandteil des bürgerlichen Haushalts, das am Familienleben partizipierte und in die patriarchalische Struktur des Hauses fest eingebunden war. Beck warb in seiner Zeitung, bei ihm sei «die Buchdruckerei und Buchhandlung ohne Lehrgeld zu erlernen»; der Besuch der lateinischen Schule genügte, und natürlich wurde eine "gute Aufführung» vorausgesetzt. ${ }^{19}$ Dass Beck Lehrlinge ausbilden und Gesellen beschäftigen konnte, spricht für den geschäftlichen Erfolg der Druckerei zumindest in den $1770 e r$ und 1780 or Jahren. Beck stieg in dieser Zeit in die städtische Honoratiorenschicht auf, die sich aus der Kauf- 


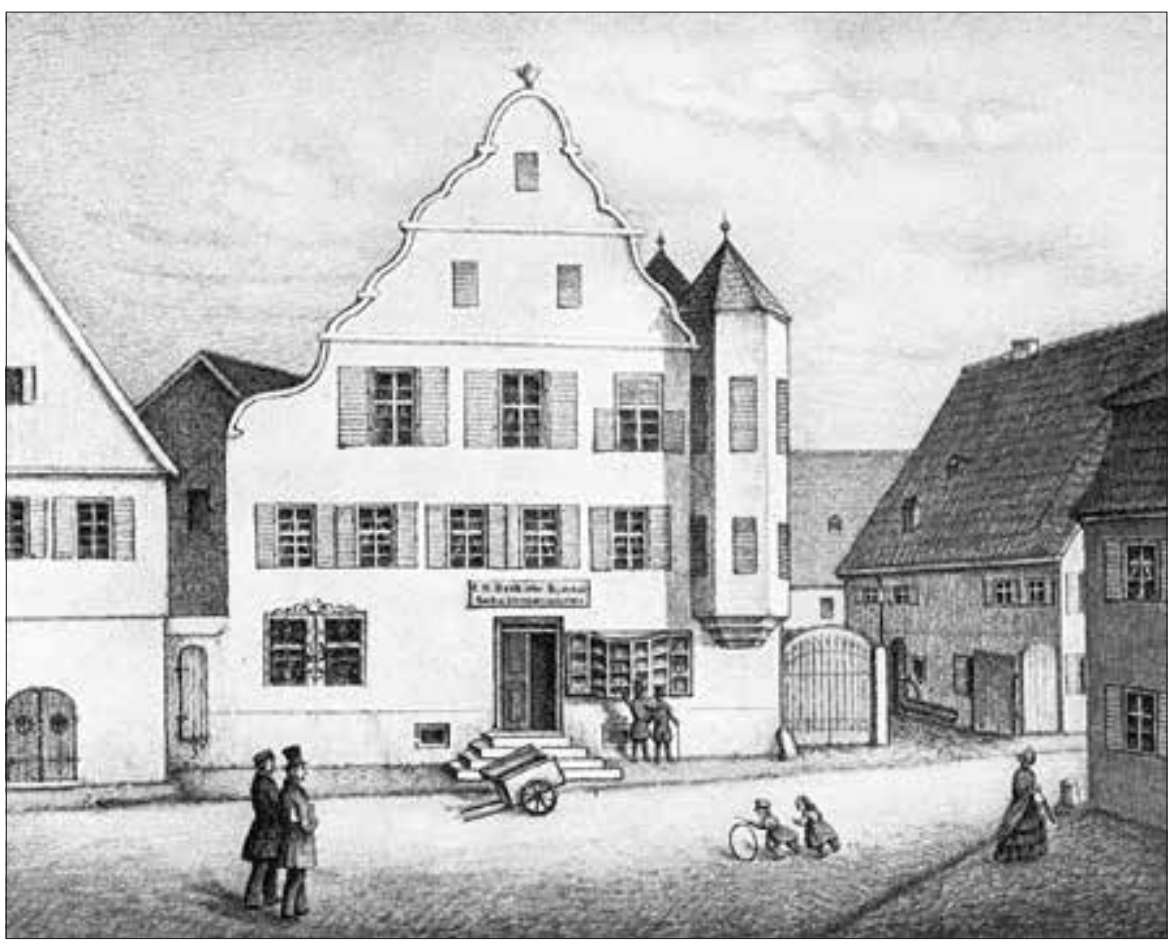

Das Stammhaus der C.H. Beck'schen Buchhandlung in Nördlingen

mannschaft rekrutierte. Sichtbares Zeichen seiner gelungenen sozialen Integration war seine Funktion als Beisitzer des städtischen Gerichts, die er seit den 179oer Jahren ausübte. ${ }^{20}$ Das Avancement war in einer Kleinstadt wie Nördlingen für einen Neubürger auch deshalb rascher möglich, weil es hier im Unterschied etwa zu Augsburg und Ulm kein vielköpfiges altes Patriziat gab, das seine überkommenen Privilegien eifersüchtig verteidigte. ${ }^{21}$

Das neue Wohnhaus in der Bergergasse bot der kinderreichen Familie Unterkunft. Der Lebensweg des Nachwuchses bestätigt, dass Beck in der Fremde mit Erfolg eine bürgerliche Existenz gegründet hatte und im Buchhandel der Stadt und Region fest verankert war. Protestantische Pflichterfüllung kennzeichnete das Leben der Eheleute Carl Gottlob und Johanna Louise. Die Ehe begann für Johanna Louise mit einer raschen Folge von Schwangerschaften. Ihr erstes Kind gebar sie 1766 mit 23, und als 1787 das fünfzehnte und letzte das Licht der Welt erblickte, war sie $44 .{ }^{22}$ Fünfzehn Töchter und Söhne waren auch in der zweiten Hälfte des 18. Jahrhunderts eine imponierende Kinderschar. Am Beispiel der Familie Beck zeigt sich 
die positive Korrelation zwischen Größe des Besitzes und Größe der Nachkommenschaft in dieser Epoche.

Die Erstgeborene (19.Januar 1766) wurde auf den Namen Heinrika Sophia Katharina getauft. Sie heiratete am 2. März 1802 den Nördlinger Barbier und Wundarzt Johann Friedrich Diehm, ${ }^{23}$ der Sohn eines Söldners des Deutschen Ordens war, in Nördlingen das Bürgerrecht erworben hatte und als Handwerker und Chirurg in der Kleinstadt wirkte, in der die Professionalisierung des Ärztestandes länger auf sich warten ließ als in den Groß- und Universitätsstädten. Am 23. August 1767 kam als zweites Kind der älteste Sohn der Familie, Carl Heinrich, zur Welt, der das väterliche Geschäft übernehmen sollte. Er musste den Buchdruck und Buchhandel nicht nur in der Provinz erlernen, sondern wurde in die Buchhandelsmetropolen Leipzig und Berlin geschickt. Auch der zweite Sohn, Johann Daniel, geboren am 31. März 1769, wählte den Buchdruck als Beruf und war von Ostern 1792 bis Pfingsten 1796 in Leipzig tätig, wo er sich «bei den Göschenschen Prachtausgaben zu Wielands Werken als ein geschickter Typograph» zeigte. ${ }^{24}$ Doch der Tod beendete früh seine vielversprechenden verlegerischen Anfänge: Johann Daniel Beck starb nur 27-jährig am 15. August 1796 in Nördlingen an Schwindsucht.

Die Tochter Rosine Luise, geboren am 3. September 1770, heiratete 1797 den Stadtammann und Pfarrerssohn Daniel Christian Stadelmeyer aus dem benachbarten Bopfingen. ${ }^{25}$ Christian Friedrich, geboren am 9. März 1773, wurde nach einer Ausbildung als Schreiber und im Rechnungswesen im württembergischen Löwenstein durch die Vermittlung seines Vaters in Nördlingen als Stiftungskassier eingestellt. ${ }^{26}$ Er zog später nach München, wo er 1819 Josephine Johanne Schertel heiratete, die Tochter eines Oberpfälzer Gutsbesitzers und Katholiken. ${ }^{27}$ Christian Friedrichs jüngere Schwester Christiane Friederike, geboren am 12. Dezember 1775, ehelichte am 31.August 1802 den Bayreuther Goldschmied und Redakteur der «Bayreuther Zeitung», Christian Heinrich Karl Burger, Sohn eines Hofkonditors. ${ }^{28}$ Dazu wurde ein Hochzeitslied gedichtet, das nach der Melodie «Ein Vogelfänger bin ich ja» aus der gut zehn Jahre zuvor in Wien uraufgeführten Mozartoper «Die Zauberflöte» vorgetragen wurde. ${ }^{29}$ Das Epithalamium integrierte traditionelle Elemente antiker und christlicher Provenienz: das Lob von Braut und Bräutigam, die Anrufung des Liebesgottes Amor, die Aufforderung zum Vollzug der Ehe und zur Zeugung zahlreicher Kinder, aber auch die Ermahnung zu ehelicher Treue. Das Gelegenheitsgedicht, das nicht den Regeln der hohen Literatur folgte, zeigt die Verwandlung dieser Gattung zu Beginn des 19. Jahrhunderts: Nicht mehr 
bedeutende Ereignisse der Politik wurden zum Thema gemacht, sondern bürgerliche Anlässe gefeiert. Aus dem öffentlichen Gedicht wurden private Verse. Die hier in Jamben vorgetragene Hoffnung auf eine zahlreiche Nachkommenschaft erfüllte sich: Unter den Kindern und Kindeskindern von Karl und Christiane Friederike Burger finden sich evangelische Theologen und Oberkonsistorialräte, die im 19. Jahrhundert die Bayerische Landeskirche in Lehre und Gottesdienst zum Vorbild des Luthertums machten. Ihr Sohn Karl August, erst Pfarrer und Dekan in Fürth, dann Oberkonsistorialrat in München, wurde auch Autor des Verlags, den sein Großvater gegründet hatte.

Heinrich Gotthelf, geboren am 26. Februar 1777, wanderte nach Basel aus und betrieb dort eine Leinwandbleicherei. Sein Sohn Heinrich Theodor begründete den Zigarrengroßhandel in der Schweiz, nachdem er 1856/57 Havanna besucht hatte. ${ }^{30}$ Wilhelmine Rosine, geboren am 4. Oktober 1784 , zog wie ihre Schwester Christiane Friederike nach Bayreuth, wo sie 1814, den verwitweten Buchdrucker Friedrich Birner heiratete; $;^{31}$ aus dieser Verbindung gingen mehrere Generationen bayerischer Pfarrersfamilien hervor. Georg August Beck, geboren am 24. August 1778, war Kaufmann und blieb bis zu seinem Tod im Jahre 1846 unverheiratet. ${ }^{32}$ Das jüngste Kind von Carl Gottlob und Johanna Louise Beck, Juliane Eberhardine, wurde am 23. März 1787 geboren. Fünf Kinder verstarben im Säuglings- oder Kindesalter. Aus dieser traurigen kleinen Schar sind mit Namen bekannt: Christoph Friedrich, geboren am 24. Oktober 1771, Johanna Maria Rosina, geboren am 4. Dezember 1779, Christian Gottlob, geboren am 31. Mai 1781, und Karoline Friederike, geboren am 17. Oktober 1785 .

Während die Familie stattlich gewachsen war, zeigten sich Ende des Jahrhunderts erste Krisensymptome, die deutlich machten, dass das Verlagsgeschäft keineswegs allen Fährnissen gewachsen war. Dem Fürsten von Oettingen-Wallerstein klagte Carl Gottlob Beck damals von «den starken Drohungen» seiner «stürmenden Gläubiger». ${ }^{35}$ Wie seine süddeutschen Mitverleger dürfte auch Beck um 1800 vor der Herausforderung gestanden haben, den bisher üblichen Tauschhandel, der gerade durch seine geistliche Kundschaft in den katholischen Konventen große Gewinne abwarf, sukzessive auf Barverkehr und Konditionshandel umzustellen. Letzterer erlaubte zwar, nicht abgesetzte Titel zurückzugeben, verkaufte Bücher konnten aber nicht mehr getauscht werden, sondern mussten in bar bezahlt werden. Doch auch die große Politik und wirtschaftliche Turbulenzen beeinträchtigten die Geschäfte. Das in Nördlingen dominierende Weberhandwerk kam zunehmend durch in- und ausländische Konkurrenz 
unter Druck. 1796 kam es zu Protesten der Weber, zu deren Niederschlagung es kaiserlicher Hilfe aus Wien bedurfte. Anlass zum Protest bot der Garnhandel, den der Bürgermeister Christian von Tröltsch seit den achtziger Jahren auf der ihm gehörenden Bleiche betrieb und der die ortsansässigen Weber ökonomisch bedrängte. ${ }^{34}$ Lastend war zudem die Zensur, die den Buchhandel mit Kurbayern, aber auch mit Württemberg in den 179oer Jahren erheblich einschränkte. ${ }^{35}$ Schließlich litt Nördlingen unter den Koalitionskriegen, die für die Stadt mit erheblichen finanziellen Aderlässen einhergingen und den Buchhandel stark beeinträchtigten. ${ }^{36}$

Als Carl Gottlob Beck am 20. Dezember 1802, nach fast 40-jähriger Tätigkeit als Buchhändler in Nördlingen, starb, hatten sich die wirtschaftlichen Verhältnisse in der Reichsstadt jedoch vorübergehend wieder stabilisiert. ${ }^{37}$ Seine Frau Johanna Louise veröffentlichte seine Todesanzeige in den «Wöchentlichen Nachrichten»: «Da es der Vorsehung gefallen, mir meinen treuen Gatten, Karl Gottlob Beck, des Stadtgerichts Beisitzer, Buchdrucker und Buchhändler allhier, nach einem dreimonatlichen schmerzhaften Krankenlager zu meinem und meiner zehn Kinder innerlichsten Schmerz heute früh halb 5 Uhr im 7o. seines Lebens und im 39. seines Geschäftsjahrs aus diesem zeitlichen zum ewigen Leben zu entziehen, so mache ich solches allen meinen Freunden, Anverwandten und Bekannten, mit der Verbittung aller Kondolenz, zu wissen. Wer des Seeligen biederes, rechtschaffenes und liebreiches Betragen gegen jedermann kannte, wird meinen gerechten Schmerz fühlen. Nördlingen, den 20. Dezember 1802. Karl Gottlob Becks seel. Witwe.»38 Sein ältester Sohn Carl Heinrich übernahm offiziell die Leitung der Verlagsbuchhandlung, führte die Geschäfte jedoch bis 1815 «auf Rechnung» seiner verwitweten Mutter. ${ }^{39}$

In seinen letzten Lebenswochen hatte Carl Gottlob Beck noch die dramatischen politischen Veränderungen in seiner Heimatstadt erlebt, die durch die Französische Revolution ausgelöst worden waren. Die Revolutionskriege hatten mit dem beispiellosen Sieg Napoleons geendet. Der Friede von Lunéville sprach die linksrheinischen Gebiete 1801 Frankreich zu, und das Reich wurde verpflichtet, die deutschen Fürsten für die verlorenen Territorien zu entschädigen. Am 25. Februar 1803 geschah dies durch den sogenannten Reichsdeputationshauptschluss, der auf dem Reichstag von Regensburg verabschiedet wurde. Man griff zu den drastischen Mitteln der Säkularisation und der Mediatisierung: Während die geistlichen Fürstentümer aufgelöst und von weltlichen Herren annektiert wurden, schlug man die reichsunmittelbaren Reichsstädte und kleinere weltliche 
Herrschaften den benachbarten größeren Fürstentümern zu. Dieser Beschluss veränderte die politische Landkarte Deutschlands radikal und hatte negative Auswirkungen für Nördlingen, das dem Kurfürstentum Bayern zufiel. $^{40}$ Bayern war für den Verlust der linksrheinischen Gebiete der Kurpfalz und des Herzogtums Jülich Kompensation zugestanden worden. De facto hatte die bayerische Herrschaft schon 1802 die alten Patriziatsverfassungen der Reichsstädte beseitigt und die Integration Nördlingens in das Kurfürstentum vorbereitet. Nach 6 oo Jahren endete die Geschichte der Freien Reichsstadt. Eine Deputation unter Führung des Bürgermeisters Christian von Tröltsch machte sich auf nach München, um dem Kurfürsten Max Joseph die schuldige Reverenz zu erweisen. Im August 1804 musste der Bürgermeister sein Amt niederlegen, was ihn allerdings nicht davon abhielt, zwei Jahre später eine große Geburtstagsfeier für Napoleon zu veranstalten, an der vielleicht auch Carl Heinrich Beck teilnahm. ${ }^{41}$

\section{Carl Heinrich Beck}

Die politischen Veränderungen hatten erhebliche wirtschaftliche Konsequenzen für die Beck'sche Verlagsbuchhandlung: Durch die Säkularisierung der Klöster und die wenig später erfolgende Mediatisierung des Fürstentums Oettingen-Wallerstein ${ }^{42}$ verlor Carl Heinrich Beck seine besten Sortimentskunden. Die geistlichen Konvente und fürstlichen Herren, die bisher großzügig geordert hatten, bestellten von heute auf morgen nichts mehr. Auch andere sichere Einnahmequellen versiegten: Die Zentralisierung der Schulpolitik und die Reorganisation der Kirchenstruktur brachten bayerische Schul- und Gesangbücher nach Nördlingen; die lokale Produktion aus dem Hause Beck erstarb. Umgekehrt konnte Carl Heinrich Beck keine Schulbücher nach Bayern absetzen, da es dort bis in die Mitte des 19. Jahrhunderts ein staatliches Monopol gab. ${ }^{43}$ In Nördlingen musste man sich damit begnügen, andernorts gedruckte Schulbücher vorrätig zu halten und an Interessenten zu veräußern. ${ }^{44}$ Damit nicht genug: Die bayerische Regierung verbot bereits am 8. März 1803 das Lotteriespiel. ${ }^{45}$ Und dann traf die in Bayern streng ausgeübte Zensur auch die Beck'sche Verlagsproduktion: ${ }^{46}$ Die im Verlag herausgegebene Zeitung, die seit 1805 «Nördlingisches Intelligenz- und Wochenblatt» und ab 1815 «Intelligenzblatt der Königlich Baierischen Stadt Nördlingen» hieß, vernachlässigte in vorauseilendem Gehorsam die «Gelehrten Anmerkungen», deren Themen die Zensurbehörde auf den Plan hätten rufen können, ver- 
stärkte das Anzeigengeschäft und informierte über Gesetze, Bekanntmachungen und Amtshandlungen. Auch die Veröffentlichung von Getreidepreisen, das wusste Carl Heinrich Beck, ließ den Zensor nicht tätig werden. Am 31.August 1804 rief das Blatt zum Besuch eines Festgottesdienstes auf, der die Einführung der bayerischen Verwaltungsorganisation feierte. ${ }^{47}$ Sukzessive richtete sich der Verlag auf das neue politische und kulturelle Zentrum aus: Im «Intelligenz- und Wochenblatt» erschienen nun häufig Hinweise auf Veranstaltungen in München, etwa die Sommer-Dult, oder es wurden das «Allerneueste Münchener Kochbuch für baierische Mädchen und Hausfrauen» und der «Baierische Nationalkalender» beworben. ${ }^{48}$

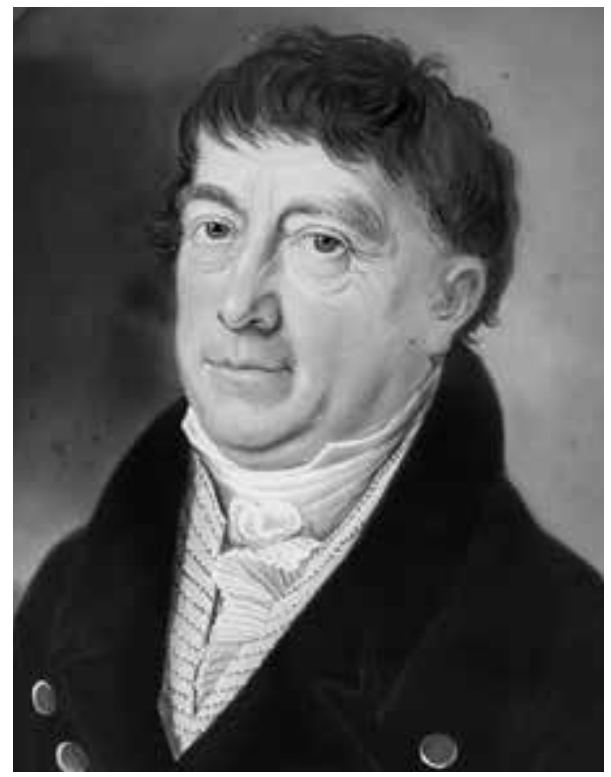

Der Verleger Carl Heinrich Beck

Als Kurfürst Max Joseph am 1. Januar 1806 zum ersten König Bayerns erhoben wurde, hatte der «baierische Buchdrucker und Buchhändler» Carl Heinrich Beck hinlänglich erfahren müssen, dass allem monarchischen Glanz zum Trotz die Zeiten schlecht waren. Wie schon die Koalitionskriege, so brachten jetzt die Napoleonischen Kriege Einschränkungen mit sich. Vom 6. zum 7. Oktober 1805 übernachtete der Kaiser der Franzosen während seines Feldzugs gegen die Österreicher in Nördlingen; wahrscheinlich zählte Carl Heinrich Beck zu den Bürgern, die französische Soldaten in ihren Häusern aufnehmen mussten. Zwei Jahre später erbrachten 1573 Nördlinger Familien 77 24,2 Quartiertage; man braucht nur wenig Phantasie, um sich die Opfer vorzustellen, die der Stadtbevölkerung dadurch abverlangt wurden. ${ }^{49}$ Angesichts der schwierigen wirtschaftlichen Lage wurden sowohl wissenschaftliche als auch literarische Werke nachgedruckt und im Nördlinger Wochenblatt angeboten. Mutter und Sohn erschlossen zudem weitere Einnahmequellen: Carl Heinrich Beck lieferte «auf Bestellung zu ganzen und halben Hunderten für Drechslerarbeiter» verschiedene Hornspitzen. «Auch sind sehr schöne ganz neue Visiten- und Gesellschaftskarten zu ganzen und halben Dutzenden von verschiedenen Dessins für billige Preise zu bekommen.» Im großen Haus vermietete man Zimmer, um zusätzliche Einnahmen zu erzielen. ${ }^{50}$ Der Verlag allein ernährte die Familie nicht mehr. 
Doch blieb man kommunikatives Zentrum von Nördlingen: In den Geschäftsräumen wurden nicht nur Bücher verkauft, als Geschenk für das Weihnachtsfest empfohlen, zur Subskription angeboten und aus der florierenden Lesebibliothek verliehen, sondern hier wurden ebenfalls Informationen über Mietwohnungen ausgetauscht, Hypothekarzinsen verhandelt und Verträge angebahnt. Auch verlorene Tabaksdosen oder Regenschirme und davongelaufene Hunde konnte man dort wiederfinden. ${ }^{51}$ Selbst ein neuer Konzertflügel aus Nussbaumholz wurde in Kommission genommen. ${ }^{52}$

Carl Heinrich Beck versuchte, Auswege aus der Krise zu finden, um den Verlag halten zu können. Er setzte auf Diversifizierung. In der späteren Verlagsgeschichtsschreibung heißt es, die damaligen Veröffentlichungen ließen «einen höheren leitenden Gesichtspunkt vermissen»; Carl Heinrich Beck habe das Unternehmen nicht «zu einem eigentlich wissenschaftlichen Verlag» weiterentwickelt. ${ }^{53}$ Damit wird die spätere Verlagspolitik zum anachronistischen Maßstab des Urteils. Es wäre demnach ein Treppenwitz der Verlagsgeschichte, dass das Unternehmen heute nicht nach dem energischen und visionären Carl Gottlob Beck, sondern nach seinem deutlich weniger begabten Sohn Carl Heinrich genannt wird.

Doch entsprach Carl Heinrich Beck nur dem Typus des reinen Provinzbuchhändlers, der zwar in seiner Heimatstadt im Magistrat saß und Landwehroffizier war, aber über den letztlich nicht viel mehr zu sagen war, als Pfarrer Johann Friedrich Schöpperlin, ein Jugendfreund, in dessen Nachruf schrieb: «Wer war zuvorkommender als Er? Wer eifriger, andern zu dienen? Wer gütiger gegen die Gehilfen seiner Offizin? Wer mehr der Armen Freund und Wohltäter? Wer mehr ein wahrer Freund und Christ wie Er?» ${ }^{54}$ Mitnichten. Der Verlag heißt zu Recht nach Carl Heinrich. Seine unternehmerisch bedeutendste Tat war der Erhalt des erst einige Jahrzehnte alten Geschäfts in widrigen Zeitläuften. Gedruckt wurde, was Absatz versprach. Genauer: Gedruckt werden musste, was Interessenten fand. Manches geplante und von dem Verleger selbst angepriesene Werk fand allerdings so wenige Subskribenten, dass es in der Verlagsgeschichte keine Spuren hinterließ. ${ }^{55}$ Vor allem verlegte Carl Heinrich Beck theologische Schriften: Predigtsammlungen und Gebetbücher dominierten sein Programm. Die wirtschaftliche Lage erlaubte keine konfessionelle Bindung: Sowohl protestantische als auch katholische Schriften wurden veröffentlicht. Die Jungfrau Maria stand im Sortiment einträchtig neben Martin Luther. ${ }^{56}$ Fortgesetzt wurde die Ratgeberliteratur: Der württembergische Pfarrer und Obstbauer Carl Vollrath Neidhart verriet «den Freunden der 
Obstkultur» die Gründe der «Herzenserleichterung eines Obstbaum-Pflanzers» (1824). Natürlich fand die Zeitgeschichte weiterhin Eingang in die Verlagsproduktion. Johann Gottfried Pahl setzte sich 1808 mit dem Rheinbund auseinander, den Napoleon aus den Trümmern des Heiligen Römischen Reiches errichtet hatte und den er als Ausgangspunkt für die staatliche und nationale Vereinigung Deutschlands interpretierte. Flammend plädierte er für die Einheit der deutschen Nation und pries den «Protektor» Napoleon überschwenglich, der Deutschland nicht unterjochen, sondern die Fürsten zu Bundesgenossen des Reiches erziehen wolle. Friedrich Wilhelm Doppelmayr sammelte 1821 die Erläuterungen und Reskripte über das bayerische Strafgesetzbuch und reflektierte die rechtliche Integration des erweiterten Königreichs Bayern. ${ }^{57}$

Carl Heinrich Beck investierte darüber hinaus in den expandierenden Markt der Kinder- und Jugendschriften und publizierte Erzählungen, die heute weitgehend unbekannte Autorinnen und Autoren wie Caroline Meinau, Johann Heinrich Meynier, Johannes Paul Pöhlmann und Aloys Adalbert Waibel (der unter dem Pseudonym Theophilus Nelk schrieb) verfassten. «Bei dem Schwalle von Jugendschriften» gebe mancher Familienvater oder Lehrer das erstbeste Buch «den zarten Pflanzen in die Hände», ohne auf dessen Qualität zu achten, klagte Carl Heinrich Beck. Seine Bücher hingegen, versicherte er zuversichtlich, dürfe man «ungescheut den Kindern überreichen», damit sie «daraus Honig saugen» könnten. ${ }^{58}$ Erziehung durch Belehrung hieß die Maxime. Doch zielten die Bücher nicht nur auf die literarisch-ästhetische Unterweisung der Kinder, sondern auch auf ihre Unterhaltung, um künftige Leser an das Haus zu binden. Zu den Jugendschriften traten Kochbücher, wie das «Neue Augsburgische Kochbuch» (1827), das nicht weniger als 956 systematisch geordnete Speisezubereitungen enthielt. 1819 von der Pfarrersgattin Sophie Juliane Weiler geschrieben, erschien es bei Beck in zweiter Auflage und erreichte, immer wieder neu bearbeitet, bis zum Jahr 1866 dreizehn Auflagen. Das Programm hatte mithin keinen hochliterarischen oder wissenschaftlichen Anspruch, sondern wollte den Namen des Nördlinger Verlags einem breiten Publikum bekannt machen. Dank Carl Heinrich Beck traten Frauen als Autorinnen auch in Bayerisch-Schwaben hervor, aber ihr Metier erstreckte sich entsprechend dem bürgerlichen Rollenverständnis auf Kinder und Küche.

Was auf den ersten Blick nach Beliebigkeit aussieht, erweist sich auf den zweiten als geschickte Überlebensstrategie. Titel, die bei einem anderen Verleger in erster Auflage erfolgreich waren, übernahm Carl Heinrich Beck in sein eigenes Programm. Begehrte Autoren konnte er nicht gewin- 
nen. Dafür war sein Honorarfonds zu schlecht ausgestattet. Überregionale Aufmerksamkeit fanden die Schriften folglich nicht. Waren in Friedrich Nicolais Lagerkatalog von 1787 noch drei Titel der Beck'schen Buchhandlung verzeichnet gewesen, so fand sich in der fünften Auflage von 1811, in der auf 273 Seiten über 5000 Titel verzeichnet waren, kein einziger Titel aus Nördlingen. Die Beck'schen Bücher waren dem Rotstift zum Opfer gefallen, weil sie zu den Veröffentlichungen zählten, die sich «entweder auf ehemalige Zeiten bezogen oder sonst nicht mehr so sehr brauchbar» waren. ${ }^{59}$ Carl Heinrich Beck hatte deutlich weniger neue Bücher zu bieten als sein Vater. Aber die Lage des Nördlinger Verlags scheint symptomatisch für die Situation des deutschen Buchhandels: Die Novitätenzahl in den Messkatalogen ging zwischen 1805 und 1817 um fast die Hälfte zurück.

Carl Heinrich Beck versuchte sich auf einem neuen Feld: dem Antiquariat. Bereits um die Jahrhundertwende hatte sich der Konditionshandel durchgesetzt, der es dem Buchhändler erlaubte, Bücher, die à condition geordert worden waren, zurückzugeben. Die Trennung zwischen Sortimenter und Verleger wurde beschleunigt, und der Zwischenhandel entstand, der die Bücher in Kommission nahm und den Sortimentern nun laufend neue Titel liefern konnte. Diese Entwicklung erleichterte auch die Entstehung des Antiquariats, durch das die unverkäuflichen Remittenden und alten Lagerbestände veräußert wurden. ${ }^{60}$ Die Säkularisation brachte nach $180 z$ zusätzlich Millionen älterer Bücher aus den Klosterbibliotheken auf den Markt, und der Nördlinger Verleger dürfte seine Verbindungen zu den katholischen Einrichtungen und dem Fürstenhaus Oettingen-Wallerstein genutzt haben, um an Bücher für das Antiquariat zu gelangen. Auch wenn dieser Geschäftszweig erst unter seinem Nachfolger aufblühte, ${ }^{61}$ zeigte Carl Heinrich Beck unternehmerisches Gespür für ein lukratives neues Geschäftsfeld im Buchhandel.

Auch die Leihbibliothek baute Carl Heinrich Beck aus. Anfang 1823 kündigte er ein «Verzeichnis der Bücher» an, «welche die Lesebibliothek der Beck'schen Buchhandlung zu Nördlingen» enthielt. ${ }^{62}$ Wir wissen nicht, welche Literatur Beck verlieh. Sicher handelte es sich nicht nur um wissenschaftliche und allgemeinbildende Werke, sondern auch um die zeittypische Unterhaltungsliteratur, die zwar oft verteufelt wurde, aber zahlreiche Leser fand. ${ }^{63}$ Die im Zuge der romantischen Mittelalterbegeisterung äußerst populären Rittergeschichten haben sicher nicht gefehlt; sie hatte Beck ohnehin in seinem Programm. ${ }^{64}$ Dann reagierte der Sortimenter Beck auf die Herausforderungen des Kolportagevertriebs, durch den ganz unterschiedliche Schriften von Hausierern an der Haustür verkauft wurden. ${ }^{65}$ Subskriptio- 
nen auf Fortsetzungslieferungen waren sehr beliebt; gehandelt wurden Lexika, Magazine, Romane, aber auch Klassiker. Als Carl Joseph Meyer, der Gründer des «Bibliographischen Instituts», seine «Miniaturbibliothek deutscher Klassiker» zu vertreiben begann, wollte Carl Heinrich Beck das Geschäft nicht den Kolporteuren überlassen, sondern nahm die Reihe in sein Sortiment: Die Edition versprach erfolgreich zu werden, denn das Bändchen kostete gerade einmal neun Kreuzer. Beck kündigte das Unternehmen im «Intelligenzblatt» am 20. April 1827 an: Müde der schlechten Übersetzungen britischer und französischer Romane, die die deutsche Leserwelt überschwemmten, sehne sich «der bessere und größere Teil des Publikums nach dem herrlichen Genuss und Besitz vaterländischer Meisterwerke», die zu einem «wohlfeilen Preis» zu erwerben seien, gleichzeitig aber «auf kostbarsten englischen Velin» gedruckt würden. Neben der Miniaturausgabe standen auch eine Kabinetts- und eine Prachtausgabe zu 18 bzw. 27 Kreuzern zur Wahl. Wer das Subskriptionsangebot nicht annahm, musste später einen um die Hälfte höheren Preis bezahlen. Den Anfang des nationalen Projekts, das auf ständeübergreifende Bildung setzte, machten Schillers Gedichte. Die «Groschenbibliothek» war auch in der schwäbischen Provinz ein Erfolg und popularisierte die zehn Jahre zuvor von Friedrich Perthes formulierte programmatische Aussage, dass der deutsche Buchhandel die «Bedingung des Daseins einer deutschen Literatur» sei. ${ }^{66}$ Mit den Kolportageromanen kamen auch die Klassiker der deutschen Literatur in das hinterste Dorf.

Wirtschaftliche Stabilität kehrte jedoch nicht durch die unternehmerische Strategie ein, sondern durch die Heirat mit einer begüterten Witwe, die über die Mittel verfügte, die Verlagsbuchhandlung finanziell zu sanieren. Am 1. Mai 1816 - ein Jahr zuvor hatte er den Verlag von seiner Mutter auf eigene Rechnung übernommen ${ }^{67}$ - ehelichte Carl Heinrich Beck, bereits 48 Jahre alt, die $3^{6}$-jährige Katharina Magdalena Zorn aus Kaufbeuren. Sie entstammte der alten und einflussreichen Kaufbeurer Familie Heinzelmann, ${ }^{68}$ aus deren Reihen erfolgreiche Handelsleute und Unternehmer hervorgegangen waren, die weit über ihre Heimatstadt hinaus bis nach Hamburg, London und Venedig, aber auch in Ungarn und in den USA gewirkt hatten; andere Mitglieder der Familie hatten das Amt des Bürgermeisters in Kaufbeuren inne. Katharina Magdalena, die im Jahr 1780 das Licht der Welt erblickt hatte, war eines von zehn Kindern, die Christian Gottlieb Heinzelmann (1755-1796) und seiner Frau Maria Elisabeth (17571793), ebenfalls eine geborene Heinzelmann, geschenkt wurden. Die Eheleute führten die beiden Hauptlinien der Familie wieder zusammen, die 
sich zu Beginn des 17. Jahrhunderts gebildet hatten. Christian Gottlieb erreichte durch seine Heirat auch die Fusion der beiden in Kaufbeuren separat existierenden Geschäfte zu dem Textilgroßhandelshaus «Gebrüder Heinzelmann». Katharina Magdalena war in erster Ehe mit dem Tierarzt und Apotheker Jakob Zorn aus Kempten vermählt gewesen, von dem sie sich jedoch hatte scheiden lassen. Zorn ging später eine zweite Ehe mit der Bankierstochter Johanna Jakobina Hillenbrand aus Augsburg ein.

Carl Heinrich Beck heiratete also eine geschiedene Frau, obwohl eine solche Verbindung im begrenzten Kosmos einer protestantischen Kleinstadt einen Skandal darstellte. Beck setzte sich darüber hinweg. Die Eheschließung begründete seine Verbindung mit dem erfolgreichen Wirtschaftsbürgertum in der knapp 150 Kilometer entfernten ehemaligen Reichsstadt. Dank der Mitgift seiner Ehefrau konnte Carl Heinrich Beck von einer zukunftweisenden Erfindung profitieren: der Steindruckerei oder Lithographie, die der Schriftsteller Alois Senefelder kaum zwanzig Jahre zuvor in München gemacht hatte. 1819 richtete Carl Heinrich Beck eine lithographische Werkstatt ein, für die sein Bayreuther Schwager die Presse konstruiert hatte. ${ }^{69}$ Stolz berichtete er in dem «Intelligenzblatt der Königlich Bayerischen Stadt Nördlingen» über die Erteilung der Konzession und warb für seine neuen Möglichkeiten. Er werde «alle kalligraphischen, tabellarischen und anderen schriftlichen Gegenstände für Königl. Stellen, Gerichtsbehörden und Ämter» liefern und alles drucken, «was ins Zeichnungsfach gehört». ${ }^{70}$ «Recht viele Aufträge» gingen ein, da er «schnell und billig» lieferte. ${ }^{71}$ Als eine der ersten Lithographien druckte er eine Reproduktion des Gemäldes «Die Belagerung von Bethulia» oder «Die Geschichte der Enthauptung des Holofernes durch Judith», das Hans Leonhard Schäufelin 1515 mit Ölfarben auf die Wand der ehemaligen Bundesstuben im Rathaus gemalt hatte und das von Friedrich Wilhelm Doppelmayr, dem späteren Bürgermeister von Nördlingen, auf Stein gezeichnet worden war. ${ }^{72}$ Die «lithographische Anstalt» erwies sich als kluge Investition in die Zukunft. Populäre Illustrationen lockerten die Jugendbücher auf, und Vorlageblätter dienten dem Schulunterricht in Kalligraphie, Zeichnen und anderen Fächern. Letztere fanden zum Teil bis in die siebziger Jahre des 19. Jahrhunderts Verwendung. ${ }^{73}$

Am 13. Februar 1834, starb Carl Heinrich Beck im siebenundsechzigsten Lebensjahr an einem Schlaganfall. Er hatte das Erbe des Vaters zunächst auf Rechnung seiner Mutter, die 1822 fast 8o-jährig gestorben war, und dann mit der tatkräftigen Unterstützung seiner Frau für seine Nachfolger bewahrt. Der unternehmerische Fokus seines Handelns blieb auf Nördlin- 
gen und Schwaben beschränkt. Er war kein Mann für überregionale Politik. An der Gründung des «Börsenvereins der Deutschen Buchhändler» wirkte er nicht mit, obschon die Vereinigung die Interessen der süddeutschen Buchhändler sowohl gegen staatliche Bevormundung als auch gegen aggressive Raubdrucke wahrnahm; Carl Heinrich Beck trat wie manch anderer Verleger und Buchhändler erst später der effizienten Standesorganisation bei, die bald nachhaltigen Einfluss auf den Buchmarkt ausüben sollte. ${ }^{74}$ Doch sein Verlag hatte an der Wende vom 18. zum 19. Jahrhundert Anteil an dem sozialen und kulturellen Modernisierungsprozess, der ganz Deutschland und Europa erfasste. Die Medienrevolution der Aufklärungszeit, die Verdichtung der Kommunikation und die Mobilisierung einer kritischen Öffentlichkeit erreichten dank seines Verlags auch die Kleinstadt Nördlingen und ihre Umgebung. Die Entwicklung des Unternehmens verlief allerdings, wie wir gesehen haben, nicht geradlinig. Wandel und Stillstand wechselten einander ab. Die Dynamik der Anfangsjahrzehnte setzte sich nicht fort. Noch war unklar, wie die $\mathrm{Zu}-$ kunft der Verlagsbuchhandlung aussehen würde. 
https://doi.org/10.17104/9783406654015-107, am 26.04.2023, 02:57:59

Open Access - (c) EY 